\title{
Relación entre la práctica deportiva en Clubes Deportivos y la mejora del control de la impulsividad en escolares
}

\section{Relationship between the practice of sports in Sports Clubs and the improvement of impulsivity control in scholars}

\section{Relação entre a prática do desporto nos Clubes Desportivos e a melhoria do controlo da impulsividade nos estudiosos}

\author{
Calleja-Reina, M. ${ }^{1}$, Rueda, J. M. ${ }^{2}$, Barbosa, A. ${ }^{3}$ \\ ${ }^{1}$ Universidad de Málaga $;{ }^{2}$ Universidad de Málaga; ${ }^{3}$ Universidad de Vigo
}

\begin{abstract}
RESUMEN
La práctica regular de Actividad Física Deportiva (en adelante AFD) tiene efectos beneficiosos a nivel físico y cognitivo. Trabajos recientes han encontrado influencia entre la práctica de AFD y la mejora en memoria, en rendimiento académico, en autoestima, en atención y en Funciones Ejecutivas.

En el presente trabajo de corte comparativo han participado 110 estudiantes de Educación Primaria y de Educación Secundaria Obligatoria de las localidades de Ourense y Málaga, con edades comprendidas entre 8 y 14 años $(\mathrm{M}=10,89 ; \mathrm{DT}=1,54)$. Los estudiantes se asignaron a diferentes grupos: estudiantes que practican AFD federados en clubes deportivos a nivel competitivo o AFD-F ( $n=37)$, estudiantes que practican AFD en horario extraescolar en los centros educativos o AFD-C $(n=37)$ y un grupo de estudiantes que no practicaban ningún tipo de AFD o No-AFD $(\mathrm{n}=36)$

Los resultados muestran la modulación de la AFD en diferentes modalidades de inhibición, comparando estudiantes que practican y los que no practican AFD (en línea con trabajos previos). Por otra parte, se ha analizado si la modalidad de AFD (AFD-F vs. AFD-C) contribuye al desarrollo diferencial de algunos componentes inhibitorios. Los resultados muestran que la AFD en ambos grupos (AFD-F y AFD-C) mejora el control atencional y el control inhibitorio, evidenciando similares resultados en la habilidad para cumplir las reglas, en la precisión en la búsqueda visual y en la flexibilidad cognitiva. Sin embargo, hemos encontrado que la práctica de AFD-F mejora los índices en el control de la impulsividad en comparación con la práctica de AFD-C.
\end{abstract}

Palabras clave: Práctica deportiva competitiva, Funciones Ejecutivas, Inhibición, Atención Selectiva, Impulsividad. 


\title{
Calleja-Reina, M., Rueda, J. M., Barbosa, A.
}

\begin{abstract}
The regular practice of Sports Physical Activity (hereinafter SPA) has beneficial effects at a physical and cognitive level. Recent works have found improvement in memory, in academic performance, in self-esteem, in attention and in Executive Functions.

In this comparative study, a sample of 110 students of Primary and Secondary Education from Ourense and Málaga (Spain) between the ages of 8 and 14 years was used $(M=10,89 ; \mathrm{SD}=1,54)$. The participants were assigned to three groups: students who practice federated physical-sports activity in sports clubs at a competitive level or SPA-F $(n=37)$, students who practice physical-sports activity in out-of-school hours in schools or SPA-C $(n=37)$ and a group of students who did not practice any type of physical-sports activity Not-SPA $(n=36)$

The results show the modulation of SPA in different inhibition modes, comparing students who do not practice SPA and those who practice SPA, in line with previous work. In a second step, we analysed whether the SPA modality, that is, whether it is developed in a sports club and therefore the students are federated (SPA -F) or whether it is developed in the school's extracurricular activities (SPA-C), contributes to the differential development of inhibition. The results show that SPA, regardless of where they carry out the activity, improves some components of EF such as attentional control, inhibitory control, showing similar results in the ability to follow the rules, in the precision in the visual search and in the cognitive flexibility. However, we have found that SPA-F practice improves impulsivity control rates compared to SPA-C practice.
\end{abstract}

Keywords: Competitive Sports Practice, Executive Functions, Inhibition, Selective Attention, Impulsivity

\section{RESUMO}

A prática regular da Actividade Física Desportiva (adiante designada por AFD) tem efeitos benéficos a nível físico e cognitivo. Na última década tem havido um interesse crescente em determinar como as capacidades cognitivas são beneficiadas pelo exercício físico, e neste sentido foram encontradas melhorias na memória, desempenho académico, auto-estima, atenção e funções executivas a partir da prática regular da AFD.

Neste estudo estudo comparativo, foi utilizada uma amostra de 110 estudantes do Ensino Primário e Secundário de Ourense e Málaga (Espanha) entre os 8 e 14 anos de idade $(\mathrm{M}=10,89$; DT=1,54). Os participantes foram distribuídos por três grupos: estudantes que praticam actividade físico-desportiva federada em clubes desportivos a nível competitivo ou AFD-F ( $\mathrm{n}=37$ ), estudantes que praticam actividade físico-desportiva em horário extra-escolar nas escolas ou AFD-C $(n=37)$ e um grupo de estudantes que não praticam qualquer tipo de actividade físico-desportiva Não AFD (n=36)

Os resultados mostram que o AFD em ambos os grupos (AFD-F e AFD-C) melhora alguns componentes do FE como o controlo atencional, o controlo inibitório, mostrando resultados semelhantes na capacidade de cumprir as regras, na precisão da pesquisa visual e na flexibilidade cognitiva. Contudo, descobrimos que a prática da AFD-F melhora os índices no controlo da impulsividade em comparação com a prática da AFD-C.

Palavras chave: Prática Desportiva Competitiva, Funções Executivas, Inibição, Atenção Selectiva, Impulsividade

\section{INTRODUCCIÓN}

Los efectos beneficiosos de la Actividad FísicoDeportiva (en adelante AFD), tanto desde el punto de vista físico como psicológico, es un hecho cada vez más evidente (OMS, 2016). Diversos estudios han puesto de manifiesto que la práctica regular de AFD produce entre quienes la realizan un aumento de la autoconfianza, un incremento en la sensación de bienestar, una mejora del funcionamiento intelectual, incluso en el rendimiento escolar ( $\mathrm{Li}$, Men, Chang, Fan, Ji, Wei, 2014, Moral, Reigal, Hernández, 2020; Reigal, Moral, Morillo, Juárez, Hernández, Morales, 2020). La relación entre AFD y el rendimiento cognitivo es un área de estudio de gran actualidad dentro de la comunidad científica (McMorris, Tomporowski, Audiffren, 2009; Páez, Reigal, Morillo, Carrasco, Hernández, Morales, 2020; Pérez, Reigal, Hernández, 2016) 


\section{Práctica deportiva y control de la impulsividad en escolares}

La cognición hace referencia a la capacidad de procesar la información procedente de los sentidos, de la experiencia previa y del conocimiento adquirido. La cognición engloba diversos procesos como la atención, la memoria, el aprendizaje, y otros procesos de alto nivel que reciben el nombre de "funciones ejecutivas" (Tirapu, Muñoz-Céspedes, Pelegrín, 2002).

Las funciones ejecutivas (FE) se han definido como procesos de orden superior que nos permiten asociar ideas simples y combinarlas para resolver situaciones de alta complejidad (Shallice, 1982), cuyo objetivo principal es facilitar la adaptación del individuo a situaciones nuevas y complejas yendo más allá de conductas habituales y automáticas (Collette, Hogge, Salmon, Van der Linden, 2006), para lo cual hace uso del control del pensamiento y de la conducta (Zelazo, Carlson, 2012). Las FE son esenciales pues en la planificación, en la organización, en la evaluación de las circunstancias para guiar y regular nuestras acciones, para adaptarnos eficazmente al entorno y para alcanzar metas (Cfr. Burgess, Simons 2005). Entre el conjunto de las FE se incluyen la resolución de problemas (razonamiento), la planificación de acción, el control inhibitorio, el control atencional (Banich, 2009; Soprano 2009), así como las habilidades que permiten establecer objetivos, planificar, iniciar actividades, autorregular $\mathrm{y}$ monitorizar las tareas, seleccionar comportamientos y conductas y ejecutar acciones para lograr los objetivos (Ardila, Pineda, Rosselli, 2000).

Existe un cierto consenso de que las principales FE son la memoria de trabajo, la flexibilidad cognitiva y la inhibición (Diamond, 2013; Davidson, Amso, Anderson, Diamond, 2006; Lehto, Juujärvi, Kooistra, Pulkkinen, 2003)

En este sentido, la inhibición se concibe como un constructo multidimensional que engloba otros procesos como el control inhibitorio, el control atencional o control de la interferencia y el control comportamental. El control inhibitorio es el que nos permite dirigir nuestra atención, nuestro comportamiento, nuestras emociones hacia la realización de la tarea, anulando los elementos distractores que nos podemos encontrar por el camino. Así, el control inhibitorio hace posible que cambiemos y que elijamos cómo reaccionar y cómo comportarnos en lugar de ser criaturas irreflexivas de hábitos (Diamond, 2013). Para algunos autores, la impulsividad es el constructo relacionado con el déficit en el control inhibitorio, y se caracteriza por la emisión de respuestas inhibitorias lentas a las tareas que debemos resolver (Logan, Schachar, Tannock, 1997).

Por su parte, el control atencional (o control de la interferencia a nivel perceptivo) nos permite atender selectivamente, centrándonos en lo que elegimos y suprimiendo otros estímulos irrelevantes para la tarea. El estudio de los componentes inhibitorios, así como los procesos atencionales, ha suscitado un especial interés por parte de los profesionales que trabajan con niños y adolescentes en el ámbito de la AFD. El motivo es que durante la práctica de $\mathrm{AFD}$, para ejecutar correctamente la tarea, es necesario poner en funcionamiento el control atencional, el control inhibitorio y el control de la interferencia (Álvarez, González, Núñez, González, Bernardo, 2007; Best, 2010; Chaddock, Hillman, Pontifex, Johnson, Raine, Kramer, 2012; Chamberlain et al., 2011; Davis et al., 2011; García, Rodríguez, Garzón, 2011; González, Campillo, Reigal, Hernández, 2018; Martín, Chirosa, Reigal, Hernández-Mendo, Juárez, Guisado, 2015; Páez el at., 2020, Parra, 2015; Reigal, Borrego, Juárez, Hernández, 2016; Reigal, Barrero, Martín, Morales, Juárez, Hernández, 2019; Reloba, Reigal, Hernández, Martínez, Martín, Chirosa, 2017; Wass, PorayskaPomsta, Johnson, 2011).

Incluso a partir de estudios con técnicas de neuroimagen se han encontrado evidencias de que la AFD practicada de forma regular favorece la maduración cerebral y el desarrollo de las redes neuronales, provocando la mejora de algunas funciones cognitivas como la atención selectiva, el control de la interferencia o la velocidad de procesamiento de los estímulos (Chaddock, Pontifex, Hillman, Kramer, 2011; Chaddock, 2012; Khan, Hillman, 2014).

El Plan Integral para la Actividad Física y el Deporte, plantea la necesidad de combinar las clases de Educación Física dentro del centro educativo con otras Actividades Físico-Deportivas (AFD) que se imparten en el marco de las clases extraescolares de centros educativos (generalmente organizadas por la Asociación de Madres y Padres de Alumnos) (AFDC) así como las que se imparte en los clubes deportivos con finalidad competitiva (AFD-F). Las exigencias en la AFD-F son mayores en número de horas, preparación física, trabajo de conductas y dedicación semanal que en la AFD-C. 


\section{Calleja-Reina, M., Rueda, J. M., Barbosa, A.}

A pesar de los trabajos previamente citados, no hemos encontrado estudios que exploren la relación entre la práctica deportiva en clubes deportivos (AFD-F) y la práctica deportiva escolar (AFD-C) y el desarrollo de determinadas funciones ejecutivas.

El primer objetivo del presente trabajo es verificar si existen diferencias en el funcionamiento ejecutivo, concretamente en control atencional, control inhibitorio y nivel de impulsividad, entre estudiantes de Educación Primaria (EP) y Enseñanza Secundaria Obligatoria (ESO) que practican AFD y los que no practican AFD, en línea con los trabajos previos. Y el segundo objetivo es comprobar si dichas diferencias en el funcionamiento ejecutivo se mantienen entre estudiantes que practican AFD a nivel competitivo, vinculados a clubes deportivos (AFD-F), aquellos estudiantes que practican AFD dentro de la oferta de actividad extraescolar en los centros educativos (AFDC) y aquellos que no practican AFD (No-AFD).

\section{MÉTODO}

Diseño

En el presente trabajo se ha planteado un estudio comparativo siguiendo un Diseño Caso Control (Ato, López, Benavente, 2013) entre estudiantes que practicaban AFD y estaban federados en clubes deportivos (AFD-F), otros que practicaban esta actividad en centros escolares (AFD-C) y un grupo que hemos llamado "de control" que no practicaban AFD (no-AFD). La selección de la muestra fue de tipo muestreo incidental.

\section{Participantes}

Participaron en la investigación 110 estudiantes que se distribuyeron de la siguiente forma. El grupo AFD-F $(n=37)$; el grupo AFD-C $(n=37)$ y el grupo no-AFD $(n=36)$. Los estudiantes procedían de Ourense y Málaga (España) y tenían edades comprendidas entre $\operatorname{los} 8$ y los 14 años $(M=10,89 ; S D=1,54)$. La AFD fue de carácter grupal, concretamente fútbol y baloncesto. El criterio de exclusión para participar en el estudio fue que hubieran repetido algún curso. En la Tabla 1 se presenta un resumen de las características de la muestra según el sexo, la edad y lugar dónde realiza la AFD.

\begin{tabular}{|c|c|c|c|c|}
\hline \multicolumn{5}{|c|}{ Datos de la muestra } \\
\hline \multirow[b]{3}{*}{ Edad } & \multicolumn{3}{|l|}{ AFD } & \multirow{4}{*}{ Total } \\
\hline & Clubes & & No & \\
\hline & Deportivos & s Colegios & realizan & \\
\hline \multirow{2}{*}{$<$ de 12 Sexo } & 12 & 10 & 6 & \\
\hline & 6 & 8 & 14 & 28 \\
\hline Total & 18 & 18 & 20 & 56 \\
\hline$\overline{>\text { de } 12}$ Sexo Masculino & 12 & 11 & 4 & 27 \\
\hline Femenino & 7 & 8 & 12 & 27 \\
\hline Total & 19 & 19 & 16 & 54 \\
\hline Sexo Masculino & 24 & 21 & 10 & 55 \\
\hline Femenino & 13 & 16 & 26 & 55 \\
\hline Total & 37 & 37 & 36 & 110 \\
\hline
\end{tabular}

En la Tabla 2 se registra el tiempo (en años) que llevaban los participantes realizando AFD.

\begin{tabular}{|lll|}
\multicolumn{3}{|c|}{ Años practicando AFD } \\
& Frecuencia & Porcentaje \\
\hline 1 año & 6 & 8,1 \\
2 años & 17 & 22,9 \\
3 años & 15 & 20,2 \\
4 años & 13 & 17,5 \\
5 años & 12 & 16,2 \\
6 años & 8 & 10,8 \\
7 años & 3 & 4,0 \\
Total & 74 & 100,0 \\
\hline Tabla 2: Distribución de participantes según el \\
número de años practicando AFD \\
\multicolumn{3}{|c}{} \\
\hline
\end{tabular}

\section{Instrumentos}

Para el presente estudio se han utilizado diversas pruebas estandarizadas

a) Test de inteligencia no verbal TONI-2A (Brown, Sherbenou, Johnsen, 2009). Esta prueba tiene como finalidad valorar la capacidad para resolver problemas con figuras abstractas, eliminando la posible influencia del lenguaje y de la habilidad motriz. 


\section{Práctica deportiva y control de la impulsividad en escolares}

b) Test de Atención d2 (Brickenkamp, 2002). Es una prueba sirve para valorar la atención selectiva y la concentración, es decir, la capacidad para atender a los estímulos relevantes de una tarea de manera rápida y precisa, ignorando los estímulos irrelevantes con un tiempo limitado.

Esta prueba proporciona información sobre la velocidad de procesamiento de los estudiantes, el cumplimiento de las instrucciones, así como de la capacidad para discriminar entre estímulos similares a nivel visual, el grado de concentración alcanzado y la motivación hacia la ejecución de la tarea.

La prueba se puede realizar de forma individual o colectiva. Consta de 47 elementos por cada una de las 14 filas. Los estímulos presentados eran $p$ o $d$ con unas rayitas que podían estar en algunas de las tres posiciones (arriba, abajo o ambas). El sujeto dispone de 20 segundos por líneas para revisar de izquierda a derecha y de arriba abajo, los elementos que coincidan con el patrón "d con dos rayitas". Esta prueba mide Total de Aciertos (TA), Total de Respuestas (TR), Tasa de errores por Omisión (O), Tasa de errores por Comisión (CO), Índice de Concentración (CON).

c) Test de las Caras-R: Test de percepción de diferencias (Thurstone, Yela, 2012). Esta prueba tiene como finalidad la evaluación de la aptitud para percibir, rápida y correctamente, semejanzas y diferencias en patrones estimulares parcialmente ordenados. Esta prueba informa de las aptitudes perceptivas, atencionales e inhibitorias de los sujetos. Esta prueba puede ser aplicada de forma individual o colectiva y consiste en determinar cuál de las tres caras representadas de forma esquemática es la diferente. La prueba consta de 60 elementos gráficos, que representan caras con trazos muy elementales (boca, ojos, cejas y pelo). La prueba recoge Aciertos (A) o número total caras tachadas correctamente; Errores (E) o número total de caras tachadas que no son correctas; Aciertos netos (A-E) o eficacia real del sujeto al penalizar los errores o las posibles respuestas al azar; Índice de Control de la Impulsividad (ICI) o nivel de control de la impulsividad que presenta el sujeto cuando realiza la tarea.

\section{Procedimiento}

Mediante correo ordinario se contactó con los centros escolares y los clubes deportivos, a fin de solicitar su colaboración en el presente trabajo. Posteriormente, se obtuvo el consentimiento de los padres o tutores legales para que los estudiantes pudieran participar, así como el permiso de la dirección de los centros escolares y clubes deportivos. Además, durante todo el proceso de investigación se respetaron los principios establecidos en la Declaración de Helsinki. Antes de la realización de las pruebas, los participantes cumplimentaron una ficha personal (Iniciales del nombre y de los apellidos, curso académico, edad y tipo de actividad físico-deportiva). Las pruebas se realizaron en diferentes días. La prueba de las Caras$\mathrm{R}$ (Test de Percepción de las Diferencias) y la prueba de Atención D2 se pasaron de forma colectiva y el mismo día con una duración de 45 minutos aproximadamente. Por su parte, la prueba de Inteligencia No Verbal TONI-2 se pasó de forma individual y en diferentes días con una duración de 20 minutos aproximadamente por cada participante. Las pruebas se pasaron en los centros escolares en un aula libre de ruidos y con buena iluminación. Siendo similares las condiciones ambientales en los clubes deportivos.

\section{Análisis de los datos}

Las variables identificadoras fueron codificadas, por ejemplo el sexo (1=hombre, $2=$ mujer); edad (se codificó en función de si los participantes tenían más de 12 años o menos de 12 años, por ser este un criterio evolutivo del desarrollo de las FE); si realizaban AFD $(1=\mathrm{Si} ; 2=\mathrm{No})$; lugar donde realiza la AFD (1= Clubes Deportivos o AFD-F; $2=$ Centros Educativos o AFDC; $3=$ No realiza AFD).

Los datos fueron analizados con la versión 25 del paquete estadístico SPPS (IBM, 2017). Para ellos se procedió a realizar una ANOVA para grupos independientes de un factor para comprobar si había diferencia entre los grupos que realizaban AFD (AFDF y AFD-C) y los que no practicaban AFD (no-AFD) en control atencional, control inhibitorio y en control de la impulsividad. A continuación, se realizaron diversas comparaciones múltiples ad hoc. La significación de cada efecto se analizó mediante comparaciones de Tukey. También se analizó la normalidad de los datos de la muestra, así como la homogeneidad de varianza de los diferentes valores mediante la prueba de Levene.

\section{RESULTADOS}

Los resultados derivados del análisis de los datos entre los grupos de estudiantes que realizan AFD y no-AFD, se presentan a continuación. En la Tabla 3 se recogen 


\section{Calleja-Reina, M., Rueda, J. M., Barbosa, A.}

los resultados del ANOVA de un factor, señalando los contrastes que resultaron significativos.

\begin{tabular}{|c|c|c|}
\hline \multicolumn{3}{|c|}{$\begin{array}{l}\text { Análisis de varianza de un factor AFD vs. No- } \\
\text { AFD }\end{array}$} \\
\hline & $\mathrm{F}_{(1,108)}$ & $\begin{array}{l}\text { Significación } \\
\text { (p) }\end{array}$ \\
\hline Total Respuesta D2 & 1.32 & $\mathrm{p}>.05$ \\
\hline Total aciertos D2 & 1.9 & $p>.05$ \\
\hline Omisiones D2 & 26.28 & $.000^{* *}$ \\
\hline Comisión D2 & 22.37 & $.000^{* *}$ \\
\hline Efectividad D2 & 0.4 & $p>.05$ \\
\hline $\begin{array}{l}\text { Índice concentración } \\
\text { D2 }\end{array}$ & 5.22 & $.000^{* *}$ \\
\hline Aciertos Test Caras & 1.37 & $\mathrm{p}>.05$ \\
\hline Errores Test Caras & 15.44 & $.000^{* *}$ \\
\hline $\begin{array}{lr}\text { Índice } & \text { Control } \\
\text { Impulsividad } & \text { Test } \\
\text { Caras } & \end{array}$ & 13.19 & $.000^{* *}$ \\
\hline $\begin{array}{l}\text { Tabla 3: Resumen resu } \\
\text { grupos (AFD vs. NO-AFl }\end{array}$ & $\operatorname{dos} \mathrm{d}$ & ANOVA entre \\
\hline
\end{tabular}

En la figura 1 se recogen las comparaciones de la tasa de errores en las diferentes tareas entre los integrantes de los grupos AFD vs. No-AFD. Se observa como los estudiantes que realizan AFD comenten menos errores comparados con los estudiantes que no realizan AFD.

En la figura 2, se representan las comparaciones en las puntuaciones de los índices entre los estudiantes que realizan AFD y los que no realizan AFD. Los estudiantes del grupo AFD muestran mayor Índice de Concentración y de Control de la Impulsividad que los estudiantes que no practican AFD.

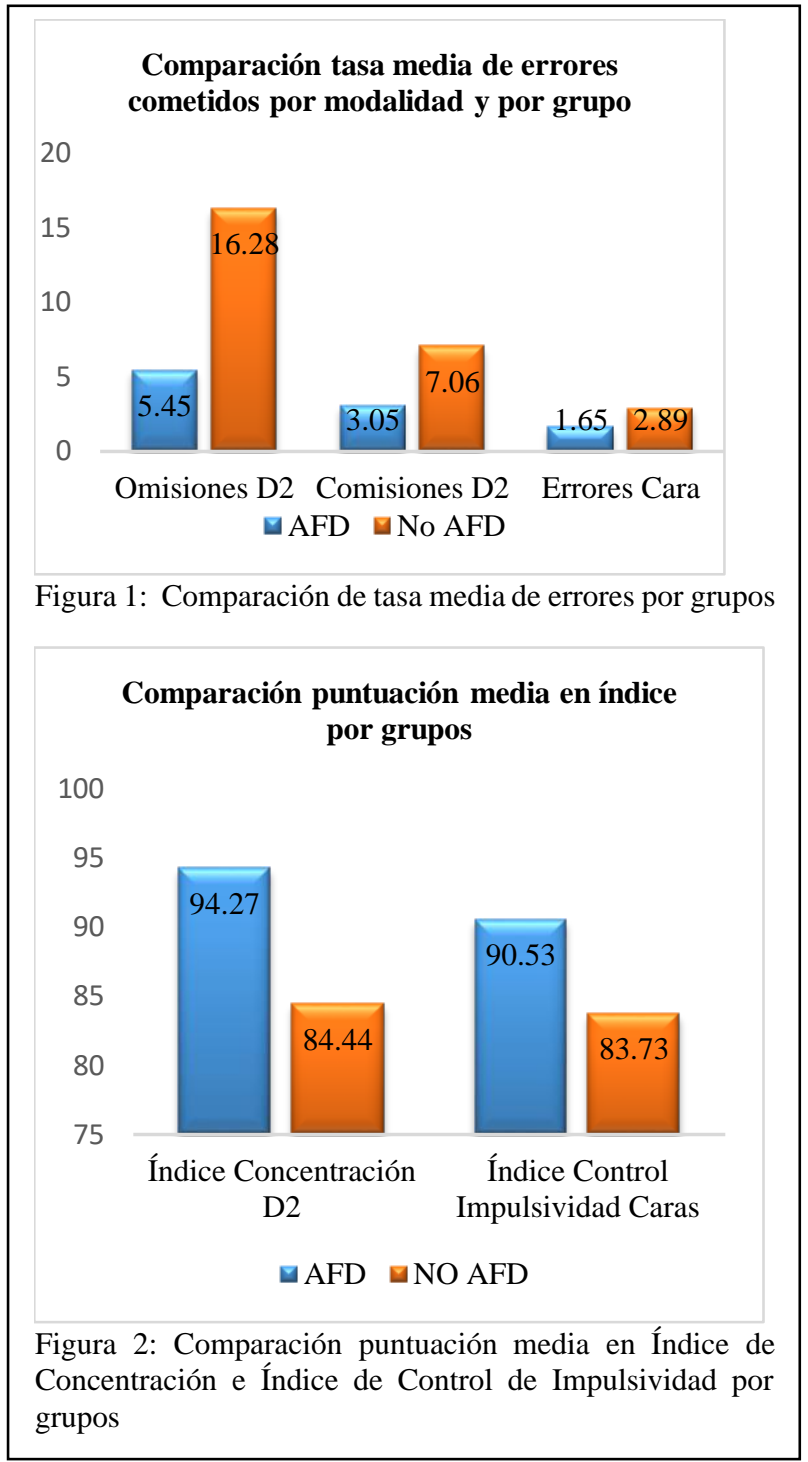

En un segundo momento se realizó una Análisis de Varianza de un solo factor para comparar si las diferencias encontradas se mantenían entre los estudiantes que realizaban AFD en función del lugar donde la practicaban dicha actividad y la modalidad de entrenamiento que realizaban. Los resultados muestran que no existen diferencias significativas entre los estudiantes de los tres grupos (AFD-F; AFD$\mathrm{C}$ y No-AFD) atribuidos ni a la edad, ni al sexo, tampoco se encuentran diferencias en la ejecución global de la tarea $\left[\mathrm{F}_{2,107}=2.46, p>.05\right]$, ni en la cantidad de elementos procesados $\left[\mathrm{F}_{2,107}=2.02, \mathrm{p}>.05\right]$, ni en la motivación hacia la realización de la tarea $\left[\mathrm{F}_{2,107}=1.00, \mathrm{p}>.05\right]$. Mientras que encontramos evidencias de un comportamiento diferencial entre los integrantes de los tres grupos en los errores cometidos, 


\section{Práctica deportiva y control de la impulsividad en escolares}

concretamente en el nivel de control atencional que presentan $\left[F_{2,107}=13.72, p=.000 * *\right]$, en la medida de control inhibitorio en la ejecución de las tareas de discriminación visual de estímulos parecidos, $\left[\mathrm{F}_{2,107}=12.17, p=.000^{* *}\right]$ y en el índice global de concentración o calidad de trabajo realizado $\left[\mathrm{F}_{2,107}=4.16, p=.018\right]$. En esa misma línea encontramos diferencias significativas entre los estudiantes de los tres grupos en la tasa de errores cometidos en la prueba de las caras $\left[\mathrm{F}_{2,107}=10.64, p=.000^{* *}\right]$ así como en el índice que valora la capacidad para controlar la impulsividad $^{1}\left[\mathrm{~F}_{2,107}=9.72, p=.000^{* *}\right]$. En la tabla 4 se recogen los contrastes significativos

\begin{tabular}{|c|c|c|}
\hline \multicolumn{3}{|c|}{$\begin{array}{l}\text { Resumen resultados Análisis de varianza de un factor } \\
\text { AFD-F, AFD-C y No-AFD }\end{array}$} \\
\hline & $F_{(2,107)}$ & $\begin{array}{l}\text { Significación } \\
(p)\end{array}$ \\
\hline Total Respuesta D2 & & $p>.05$ \\
\hline Total aciertos D2 & & $p>.05$ \\
\hline Omisiones D2 & 13.72 & $.000^{* *}$ \\
\hline Comisión D2 & 12.17 & $.000^{* *}$ \\
\hline Efectividad D2 & & $p>.05$ \\
\hline Índice concentración D2 & 4.16 & $.000^{* *}$ \\
\hline Aciertos Test Caras & & $p>.05$ \\
\hline Errores Test Caras & 10.64 & $.000^{* *}$ \\
\hline $\begin{array}{l}\text { Índice Control Impulsividad } \\
\text { Test Caras }\end{array}$ & 9.72 & $.000^{* *}$ \\
\hline
\end{tabular}

En la figura 3 se representa la comparación en la tasa de errores que han alcanzado los integrantes de los tres grupos de estudiantes. En ese sentido observamos que los estudiantes que practica AFD-F cometen menos errores en las tres modalidades de errores analizadas (omisión, comisión y búsqueda de caras), seguidos por los que practican AFD-C y los que más errores cometen en tareas de búsqueda visual son los estudiantes que no practican AFD.

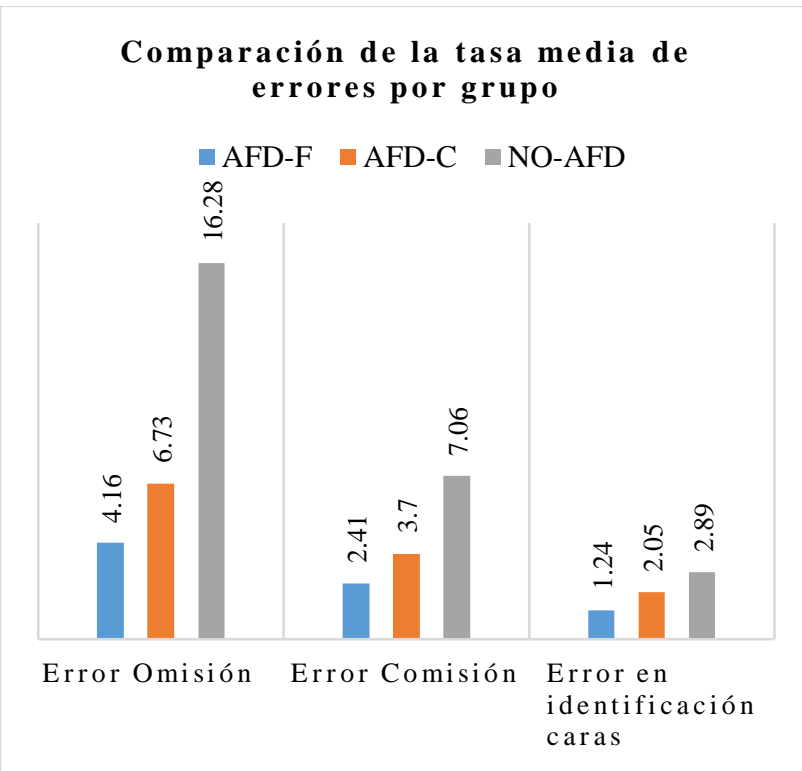

Figura 3: Comparación tasa media de errores según los grupos

En la figura 4 se representa la distribución de las puntuaciones alcanzadas en los diferentes índices (Concentración y Control de la Impulsividad) por grupos de participantes. Los resultados muestran que los estudiantes del grupo AFD-F obtienen mejor puntuación en concentración y en control de Impulsividad, seguidos de los del grupo AFD-C y por último los del grupo no-AFD muestran peores resultados en dichos índices.

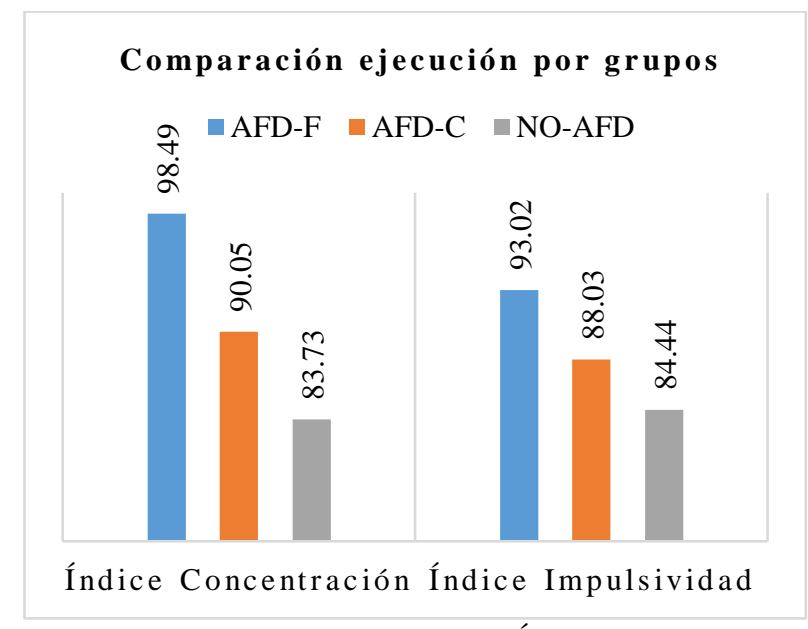

Figura 4: Comparación de valores de los Índices por grupos

\footnotetext{
${ }^{1}$ Entendida déficit en el control inhibitorio (Logan et al., 1997)
} 


\section{Calleja-Reina, M., Rueda, J. M., Barbosa, A.}

Del análisis post hoc, a partir de comparaciones múltiples, con la prueba HSD Tukey se desprende que los estudiantes que realizan AFD-F muestran mejor ejecución, seguidos por los que realizan AFD-C y por último estarían los que no realizan AFD.

Analizando pormenorizadamente el tipo de errores cometidos, observamos en la Figura 5, que los estudiantes vinculados a clubes deportivos registraban una tasa media menor de errores por omisión $(4,16)$, seguidos por los estudiantes de centros escolares que practicaban AFD $(6,73)$ y por último estarían los estudiantes que no practicaban ninguna $\operatorname{AFD}(16,28)$. Esta modalidad de errores por omisión informa del control atencional que activan los participantes al elegir la respuesta correcta, es decir, al diferenciar entre dos estímulos presentados a nivel visual muy parecidos. Los resultados de los análisis post hoc arrojaron diferencias significativas en control atencional entre estudiantes que practicaban AFD-F y no AFD $\left[t_{73}=-12.11, p=.000 * *\right], y$ entre los que practicaban AFD-C y los que no practicaban AFD $[t$ $\left.{ }_{73}=-9.54, p=.000^{* *}\right]$. Los dos grupos de estudiantes que realizaban AFD, independientemente del lugar donde lo practicaban, no mostraron diferencias significativas en la capacidad para cumplimiento de las reglas ni en la precisión de la búsqueda $\left[t_{74}=-2.56\right.$, $p>.05]$.

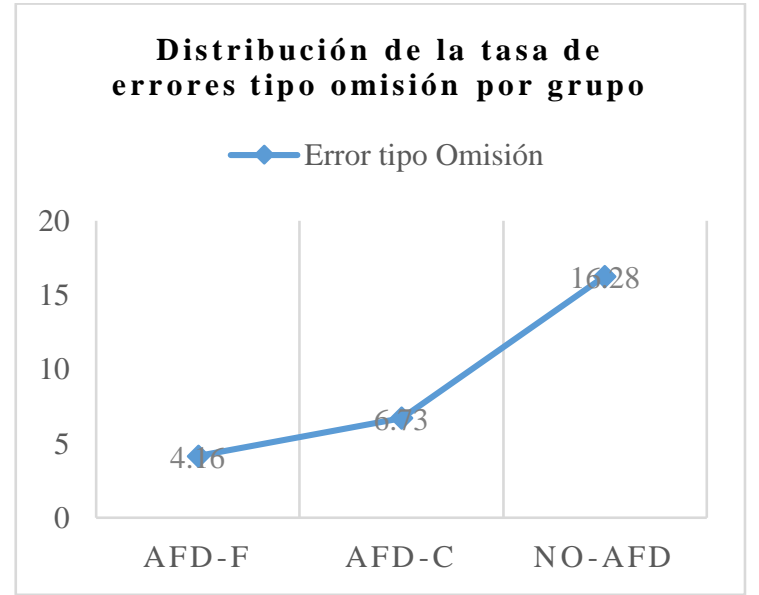

Figura 5: Distribución tasa errores tipo Omisión por grupo

En cuanto al grado de control inhibitorio que realizan los participantes obtenido a partir del análisis post hoc de los errores por comisión, se han encontrado diferencias significativas entre los grupos de estudiantes que practicaban AFD y los que no practicaban AFD. Concretamente los estudiantes del grupo AFD-F cometían menos errores de la modalidad "comisión" que los del grupo no-AFD $\left[t_{73}=-4.65\right.$, $\left.p=.000^{* *}\right)$. Y los estudiantes del grupo AFD-C mostraban un patrón inhibitorio significativamente diferente al de los estudiantes que no realizaban AFD $\left[\begin{array}{l}t \\ 73\end{array}=-3.35, p=.002\right]$. La comparación en el rendimiento de los dos grupos que practicaban AFD (AFD-F y AFD-C) no reflejó diferencias significativas $\left[t_{74}=-1.29, p>.05\right]$. En otras palabras, en la Figura 6 se observa como la tasa de error por comisión en los estudiantes AFD-F fue menor $(2,41)$, seguidos por estudiantes AFD-C $(3,70)$ y por último estarían los estudiantes que no practicaban ninguna $\operatorname{AFD}(7,06)$, aunque entre los dos primeros estas diferencias no llegaron a ser significativa.

\section{Distribución de la tasa de Errores tipo Comisión por grupo}

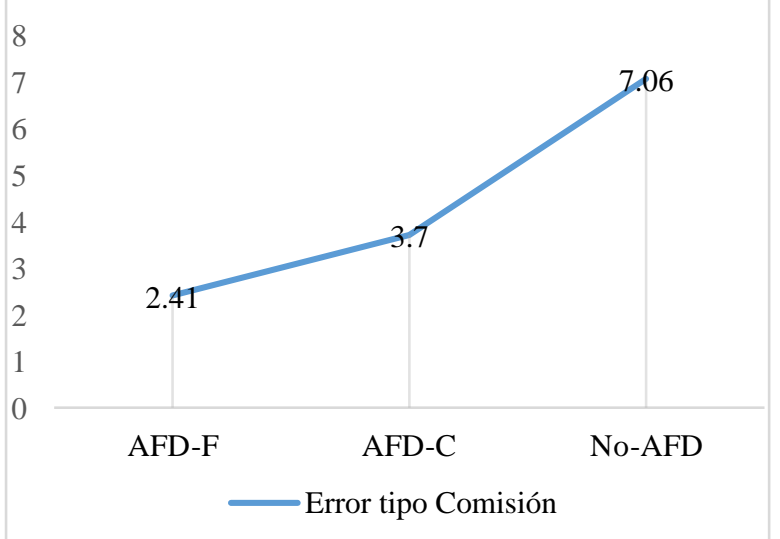

Figura 6: Distribución tasa errores tipo Comisión por grupo

En cuanto a la medida que informa sobre la calidad del trabajo realizado, del análisis post hoc se desprende que existen diferencias significativas entre los estudiantes que realizan AFD-F y los que no realizan $\operatorname{AFD}\left[t_{73}=14.04, p=.015^{*}\right]$. No existiendo diferencias entre AFD-F y AFD-C [ $\left.t_{74}=8.43, p>.05\right]$, ni entre AFD-C y no AFD $\left[t_{73}=5.61, p>.05\right]$. En general, los participantes de AFD-F obtienen un mejor rendimiento global en la tarea de buscar estímulos similares $(98,9)$, seguido por los estudiantes del grupo AFD-C $(90,05)$ y por último el peor rendimiento lo 


\section{Práctica deportiva y control de la impulsividad en escolares}

obtendrían los estudiantes del grupo no-AFD $(84,4)$, aunque las diferencias significativas se encuentran entre los grupos AFD-F y no-AFD (Ver Figura 7).

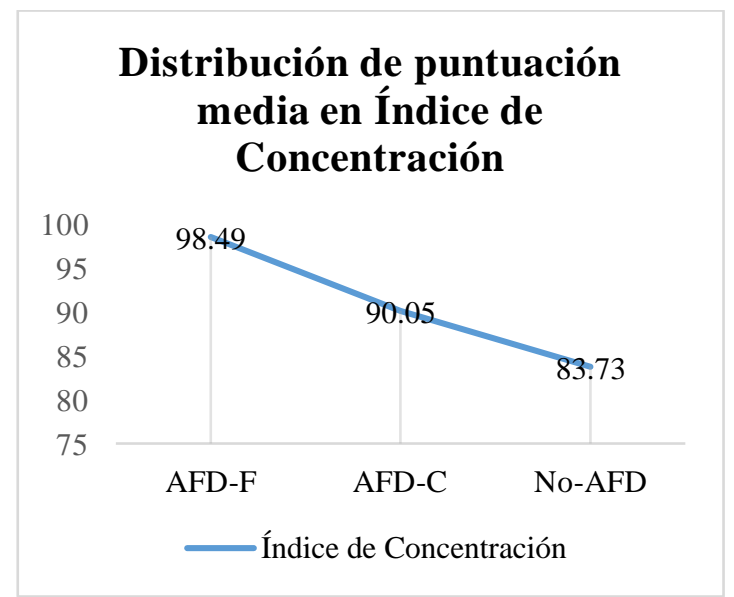

Figura 7: Índice de nivel de concentración por grupos

Por otro lado, a la vista de los resultados de las pruebas post hoc observamos que existen diferencias significativas en la tasa de errores cometidos en la tarea de identificación de caras entre estudiantes del grupo AFD-F y no-AFD [ $\left.\mathrm{t}_{73}=-1.64, \mathrm{p}=.000^{* *}\right]$. Los estudiantes que realizan AFD en clubes deportivos registran menos errores en tareas de identificación de caras que los que no realizan AFD. Sin embargo, no encontramos diferencias entre las otras comparaciones posibles (AFD-F y AFD-C, ni entre AFD-C y noAFD). La distribución de la tasa de errores media cometidos se recoge en la figura 8 .

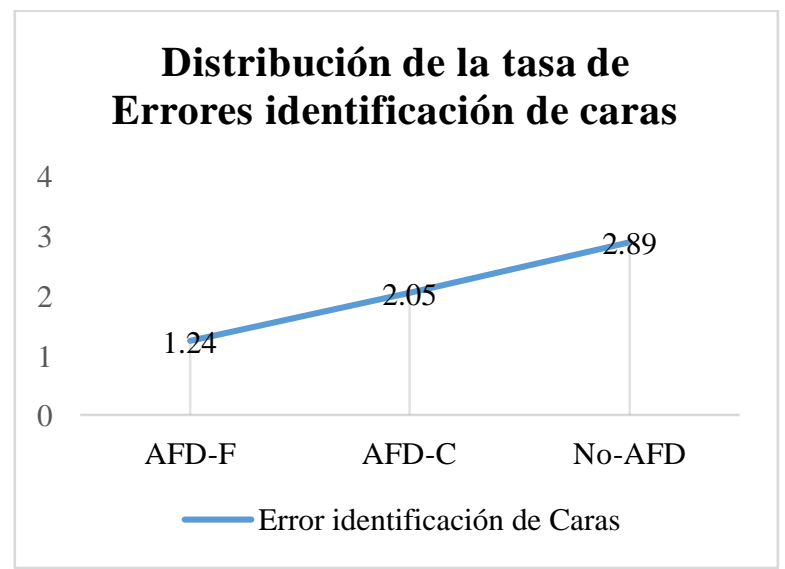

Figura 8: Distribución tasa de errores identificación de caras
En cuanto al control de la impulsividad encontramos diferencias significativas en las puntuaciones obtenidas por los estudiantes de los tres grupos. Concretamente hemos observado un comportamiento diferencial entre estudiantes del grupo AFD-F y los de no-AFD $\left[\mathrm{t}_{73}=9.28, \mathrm{p}=.000 * *\right]$ y entre estudiantes de los grupos AFD-F y AFD-C [ $\left.\mathrm{t}_{74}=4.98, \mathrm{p}=.049\right]$. De lo que se desprende que los estudiantes que practican AFD en los clubes deportivos muestran mayor nivel de control de impulsividad conceptualizada como un menor déficit en el control inhibitorio. En la Figura 9 se recoge las puntuaciones en el índice de control de la impulsividad en los tres grupos.

\section{Puntuación media en Índice de Control de Impulsividad por grupos}

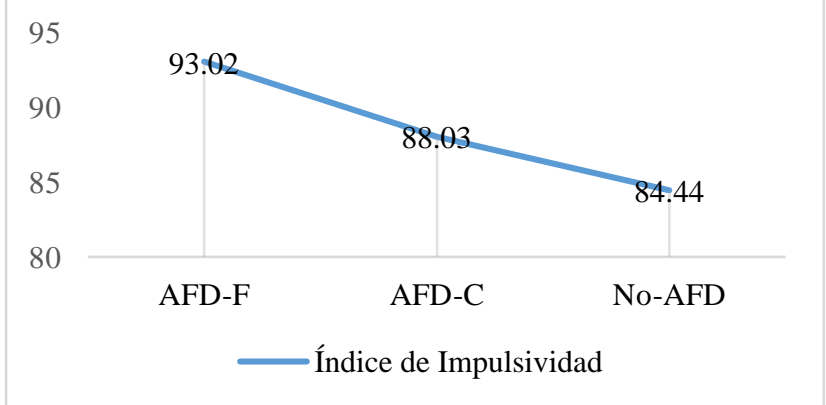

Figura 9: Puntuaciones media Índice de Control de Impulsividad por grupos

\section{DISCUSIÓN}

Las FE son esenciales en los procesos de planificación, organización, evaluación de las circunstancias que guían nuestras acciones (Burgess, Simons 2005). Existe un extenso corpus de evidencias que señalan la relación positiva entre la AFD realizada de forma regular y la mejora de algunas FE (Best, 2010; Chaddock et al., 2012; Martín et al., 2015; Reigal et al, 2020, entre otros). Sin embargo, no se han encontrado estudios que analicen si la modalidad de AFD (en clubes deportivos o en centros escolares) contribuye significativamente a la mejora de las habilidades cognitivas relacionadas con la atención y con la inhibición

La finalidad del presente trabajo era analizar los efectos de la práctica AFD en el componente 


\section{Calleja-Reina, M., Rueda, J. M., Barbosa, A.}

atencional e inhibitorio del funcionamiento cognitivo en estudiantes de EP y ESO.

El primer objetivo fue comprobar si existían diferencias entre grupos de escolares que practicaban AFD y un grupo de escolares que no practicaban AFD en el funcionamiento atencional e inhibitorio, en línea con los trabajos previos (González et al, 2018; Moral et al, 2020; Reigal et al, 2019).

Los resultados han puesto de manifiesto la existencia de diferencias entre estudiantes que practican AFD y los que no practicaban AFD en los componentes atencionales e inhibitorios. Concretamente, los alumnos que practican AFD muestran una menor tasa de errores en tareas de tipo perceptivo, mejores niveles de control (tanto atencional como inhibitorio) y mejor puntuación en los índices de concentración, frente a los estudiantes de la misma edad que no practican AFD. Estos hallazgos están en consonancia con trabajos previos en los que se valora la práctica de actividad física y el funcionamiento ejecutivo, la atención selectiva y la concentración (Guiney, Machado, 2013; Pérez et al., 2016; Reigal et al., 2016, Reigal et al, 2019). Sin embargo, no se han encontrado diferencias ni en la velocidad de procesamiento, ni en la cantidad de trabajo realizado entre los grupos estudiados. Este dato puede ser explicado por la elevada motivación hacia la tarea que mostraron todos los participantes en el estudio, aunque la tendencia en la ejecución global fuese mejor para los que practicaban AFD.

Un dato que ha resultado relevante ha sido el hecho de encontrar diferencias significativas entre los grupos que practicaban y los que no practicaban AFD en el índice de control de la impulsividad. Concretamente, los integrantes del primer grupo presentan mejores niveles en control de impulsividad que los del segundo.

A modo de conclusión de los datos referidos al primer objetivo, podemos sostener que los estudiantes que realizan AFD, independientemente de la modalidad e intensidad del entrenamiento, cuentan con buena habilidad para cumplir las reglas, son precisos en los procesos de búsqueda visual, disponen de buen nivel de control de la impulsividad, todos ellos componentes esenciales de las FE.

Para constatar si dichas diferencias en funcionamiento ejecutivo se mantenían entre los tres grupos de estudiantes se procedió a realizar un análisis post hoc. Los resultados arrojan diferencias significativas en funcionamiento cognitivo entre los integrantes del grupo AFD-F y los integrantes del grupo no-AFD en todas las modalidades de control (atencional e inhibitorio), así como en los índices de concentración y en el de control de la impulsividad.

Por otra parte, dentro del segundo objetivo se pretendía determinar si el lugar donde se realizaba la AFD, y por ende la modalidad y la intensidad del entrenamiento, tenía un efecto diferencial en el desarrollo de diversos componentes atencionales e inhibitorios de las FE.

Aunque la tendencia de los datos sugiere una mejor ejecución en las tareas atencionales e inhibitorias de los estudiantes del grupo AFD-F en comparación con los estudiantes del grupo AFD-C, sin embargo, los resultados de las comparaciones múltiples HSD Tukey no arrojan diferencias significativas entre ambos grupos ni en control atencional, ni en control inhibitorio, ni en control ejecutivo, ni en el índice de concentración, ni en los errores en la tarea de reconocimiento de caras. Estos datos pueden ser explicados por el hecho de que ambos grupos de escolares realizaban AFD. Y, en línea con los trabajos recientes, la AFD mejora el componente atencional e inhibitorio como parte de las FE (Páez et al., 2020; Reigal, et al, 2019; Reigal et al, 2020), independientemente del nivel de exigencia diferencial entre las modalidades de AFD (en clubes deportivos vs. en centros escolares).

Sin embargo, en el presente trabajo se han encontrado diferencias significativas en el índice de control de la impulsividad de la prueba de las caras entre los grupos de estudiantes que practican AFD. Los estudiantes de los clubes deportivos desarrollan mayor control de la impulsividad en comparación con los estudiantes que realizan la AFD en centros escolares, en el marco de las actividades extraescolares. El índice de control de impulsividad informa del déficit en control inhibitorio y refleja el estilo cognitivo dentro del gradiente impulsivo-reflexivo. Las elevadas puntuaciones medias, cercanas a 100, informan de que el sujeto presenta un adecuado control de la impulsividad, realizando la tarea de una forma reflexiva $y$ cometiendo pocos errores. Por el contrario, las puntuaciones más bajas en esta prueba reflejan un estilo cognitivo más impulsivo, realizando la tarea de emitir un juicio sobre semejanzas vs. diferencias entre estímulos presentados de manera poco reflexiva, lo que se traduce en mayor tasa de errores y por tanto en una falta de control inhibitorio. 


\section{Práctica deportiva y control de la impulsividad en escolares}

En conclusión, podemos sostener que en general la práctica de AFD tiene efectos positivos sobre los componentes de las FE relacionados con el control atencional, el control inhibitorio, la capacidad de concentración y el control de la impulsividad de los estudiantes. Pero, de nuestros datos se desprende además que la práctica deportiva en clubes deportivos caracterizada por una mayor exigencia en el número de horas/semanas, una mayor preparación física, así como el trabajo sobre rutinas conductuales en escolares, contribuye a adquirir hábitos relacionados con la capacidad de dirigir los recursos atencionales hacia la tarea mejorando el control de la impulsividad, incrementando pues el control inhibitorio.

En futuros estudios se podría ampliar el tamaño de la muestra, así como explorar si estos datos se mantienen con otras modalidades de AFD, a fin de obtener resultados más robustos.

\section{APLICACIONES PRÁCTICAS}

A modo de conclusión, los resultados encontrados en esta investigación aportan nuevos datos sobre la relación entre la práctica de AFD y el desarrollo de las FE en alumnos/as de EP y ESO. La práctica de AFD en clubes deportivos contribuye al desarrollo de la capacidad inhibitoria, componente de las FE.

En futuras investigaciones, se podría aumentar la muestra para obtener datos más robustos.

\section{REFERENCIAS}

1. Álvarez, P., González-Castro, P., Núñez, J. C., González-Pineda, J., Bernardo, A. (2007). Evaluación y control de la activación cortical en el déficit de atención sostenida. Internacional Journal of Clinical and Health Psychology, 8, 509524.

2. Ardila, A., Pineda, D., Rosselli, M. (2000). Correlation Between Intelligence Test Scores and Executive Function Measures. Archives of Clinical Neuropsychology, 15(1), 31-36. https://doi.org/10.1093/arclin/15.1.31

3. Ato, M., López, J.J., Benavente, A. (2013). Un sistema de clasificación de los diseños de investigación en psicología. Anales de Psicología, 29(3), 1038-1059. Disponible en: https://www.redalyc.org/articulo.oa?id=167/1672 8244043
4. Banich, M.T. (2009). Executive Function: The search for an integrated account. Current Directions in Psychological Science, 18(2), 89-94. https://doi.org/10.1111/j.14678721.2009.01615.x

5. Best, J.R. (2010). Effects of physical activity on children's executive function: Contributions of experimental research on aerobic exercise. Developmental Review, 30(4), 331-351. https://doi.org/10.1016/j.dr.2010.08.001

6. Brickenkamp, R. (2002). D2. Test de atención. Madrid: TEA Ediciones.

7. Brown, L., Sherbenou, R.J., Johnsen, S.K. (2009). TONI 2. Test de Inteligencia No Verbal. Madrid: TEA Ediciones.

8. Burgess, P. W., Simons, J. S. (2005). 18 Theories of frontal lobe executive function: clinical applications. In: Halligan, PW.; Wade, DT., editors. Effectiveness of Rehabilitation for Cognitive Deficits. Oxford Univ. Press; New York, pp. 211-31. https://doi.org/10.1093/acprof:oso/97801985265 44.003.0018

9. Chaddock, L., Hillman, C.H., Pontifex, M.B., Jonhson, C.R., Raine, L.B., Kramer, A.F. (2012). Childhood aerobic fitness predicts cognitive performance one year later. Journal of Sport Sciences, 30(5), 421-430. https://doi.org/10.1080/02640414.2011.647706

10. Chaddock, L., Pontifex, M. B., Hillman, C. H., Kramer, A. F. (2011). A review of the relation of aerobic fitness and physical activity to brain structure and function in children. Journal of the International Neuropsychological Society, 17, 975-985. Doi: http://dx.doi. org/10.1017/s1355617711000567

11. Chamberlain, S. R., Robbins, T. W., WinderRhodes, S., Müller, U., Sahakian, B. J., Blackwell, A. D., Barnett, J.H. (2011). Translational approaches to frontostriatal dysfunction in attention-deficit/hyperactivity disorder using a computerized neuropsychological battery. Biological Psychiatry, 69, 1192-1203. https://doi.org/10.1016/j.biopsych.2010.08.019

12. Collette, F., Hogge, M., Salmon, E., Van der Linden, M. (2006). Exploration of the neural substrates of executive functioning by functional 


\section{Calleja-Reina, M., Rueda, J. M., Barbosa, A.}

neuroimaging. Neuroscience, 139, 209-221. https://doi.org/10.1016/j.neuroscience.2005.05.0 35

13. Davidson, M. C., Amso, D., Anderson, L. C., Diamond, A. (2006). Development of cognitive control and executive functions from 4 to 13 years: Evidence from manipulations of memory, inhibition, and task switching. Neuropsychologia, 44(11), 2037-2078. https://doi.org/10.1016/j.neuropsychologia.2006. 02.006

14. Davis, C.L., Tomporowski, P.D., McDowell, J E., Austin, B.P., Miller, P.H., Yanasak, N.E., Naglieri, J.A. (2011). Exercise improves executive function and achievement and alters brain activation in overweight children: a randomized, controlled trial. Health psychology: Official Journal of the Division of Health Psychology, American Psychological Association, 30(1), 91-98. https://doi.org/10.1037/a0021766

15. Diamond, A. (2013). Executive functions. Annual review of psychology, 64, 135-168. https://doi.org/10.1146/annurev-psych-113011143750

16. García, S., Rodríguez, A., Garzón, A. (2011). Conceptualización de inteligencia táctica en fútbol: consideraciones para el desarrollo de un instrumento de evaluación en el campo desde las funciones ejecutivas. Cuadernos de Psicología del Deporte, 11, 69-78.

17. González, F. D., Campillo, L. M., Reigal Garrido, R., Hernández Mendo, A. (2018). Condición física y atención selectiva en una muestra preadolescente/Physical fitness and selective attention in a preadolescent sample/Condição física e atenção seletiva em uma amostra préadolescente. Cuadernos de Psicología del Deporte, 18(2), 33-42.

18. Guiney, H., Machado, L. (2013). Benefits of regular aerobic exercise for executive functioning in healthy populations. Psychonomic Bulletin \& Review, 20(1), 73-86. https://doi.org/10.3758/s13423-012-0345-4

19. IBM Corp. Released 2017. IBM SPSS Statistics for Windows, Version 25.0. Armonk, NY: IBM Corp.
20. Khan, N., Hillman, C. H. (2014). The Relation of Childhood Physical Activity and Aerobic Fitness to Brain Function and Cognition: A Review. Pediatric Exercise Science, 26, 138-146. Doi: http://dx.doi.org/10.1123/pes.2013-0125.

21. Lehto, J. E., Juujärvi, P., Kooistra, L., Pulkkinen, L. (2003). Dimensions of executive functioning: Evidence from children. British Journal of Developmental Psychology, 21(1), 59-80. https://doi.org/10.1348/026151003321164627

22. Li, L., Men, W. W., Chang, Y. K., Fan, M. X., Ji, L., Wei, G. X. (2014). Acute aerobic exercise increases cortical activity during working memory: a functional MRI study in female college students. PloS one, 9(6), e99222. https://doi.org/10.1371/journal.pone.0099222

23. Logan, G.D., Schachar, R.J., Tannock, R. (1997). Impulsivity and Inhibitory Control. Psychological Science, 8(1), 60-64. doi:10.1111/j.14679280.1997.tb00545.x https://doi.org/10.1111/j.14679280.1997.tb00545.x

24. Martín, I., Chirosa, L.J., Reigal, R., Hernández Mendo, A., Juárez, R., Guisado, R. (2015). Efectos de la actividad física sobre las funciones ejecutivas en una muestra de adolescentes. Anales de la Psicología, 31(3), 962-971. https://doi.org/10.6018/analesps.31.3.171601

25. McMorris, T., Tomporowski, P., Audiffren, M. (2009) Exercise and cognitive function. Michigan: Wileyhttps://doi.org/10.1002/9780470740668

26. Moral Campillo, L., Reigal Garrido, R. E., Hernández Mendo, A. (2020). Actividad física, funcionamiento cognitivo y psicosocial en una muestra preadolescente. Revista de Psicología del Deporte, 29(1), 0123-132.

27. Organización Mundial de la Salud (2016). Informe de la Comisión para acabar con la obesidad infantil. Recuperado https://www.who.int/end-childhoodobesity/news/launch-final-report/es/. Abril 2020

28. Páez Maldonado, J. A., Reigal, R. E., Morillo, J. P., Carrasco, H., Hernández, A., Morales, V. (2020). Physical Fitness, Selective Attention and Academic Performance in a Pre-Adolescent Sample. International Journal of Environmental 


\section{Práctica deportiva y control de la impulsividad en escolares}

Research and Public Health, 17(17), 6216. https://doi.org/10.3390/ijerph17176216

29. Parra, L. (2015) Impacto de la actividad física en el desarrollo y evolución de las funciones ejecutivas. Actualizaciones en Psicología Integrativa (AcPI), 3, 86-94.

30. Pérez Lobato, R., Reigal, R., Hérnandez, A. (2016). Relaciones entre la práctica física, condición física y atención en una muestra adolescente. Revista de Psicología del Deporte, 25 (1), 179-186.

31. Reigal Garrido, R. E., Borrego, J. L., Juárez, R., Hernández Mendo, A. (2016). Práctica física regular y funcionamiento cognitivo en una muestra de adolescentes. Revista Iberoamericana de Psicología del Ejercicio y el Deporte, 11(2), 201-209.

32. Reigal Garrido, R. E., Barrero, S., Martín, I., Morales Sánchez, V., Juárez, R., Hernández Mendo, A. (2019). Relationships between reaction time, selective attention, physical activity and physical fitness in preteens. Frontiers in Psychology, $10, \quad 2278$. https://doi.org/10.3389/fpsyg.2019.02278

33. Reigal, R. E.; Moral Campillo, L.; Juárez Ruiz de Mier, R.; Morillo Baro, J. P.; Morales Sánchez, V.; Pastrana, J. L., Hernández-Mendo, A. (2020). Physical fitness level is related to attention and concentration in adolescents. Frontiers in Psychology, 11, 110. https://doi.org/10.3389/fpsyg.2020.00110

34. Reloba Martínez, S., Reigal, R. E., Hernández Mendo, A., Martínez López, E. J., MartínTamayo, I., Chirosa Ríos, L. J. (2017). Effects of vigorous extracurricular physical exercise on the attention of schoolchildren. Revista de Psicología del Deporte, 26, 29-36.

35. Shallice, T. (1982). Specific impairments of planning. Philosophical transactions of the Royal Society of London. Series B, Biological sciences, 298, 199-209. https://doi.org/10.1098/rstb.1982.0082

36. Soprano, A.M. (2009). Evaluación de las funciones ejecutivas. Revista de Neurología, $37(1)$, 44-50. https://doi.org/10.33588/rn.3701.2003237
37. Thurstone, L.L., Yela, M. (2012). CARAS-R. Test de percepción de diferencias. Madrid: TEA Ediciones.

38. Tirapu J., Muñoz-Céspedes J.M., Pelegrín C. (2002). Funciones ejecutivas: necesidad de una integración conceptual. Revista de Neurología, 34 , $673-85$. https://doi.org/10.33588/rn.3407.2001311

39. Wass, S., Porayska-Pomsta, K., Johnson, M. H. (2011). Training attentional control in infancy. Current Biology, 21, 1543-1547. https://doi.org/10.1016/j.cub.2011.08.004

40. Zelazo, P.D., Carlson, S. (2012). Hot and Cool Executive Function in Childhood and Adolescence: Development and Plasticity. Child Development Perspectives, 6 (4) 354-360. https://doi.org/10.1111/j.1750-606.2012.00246.x 